

\title{
Demonstrating full integration process for electroactive polymer microtransducers to realize soft microchips
} Lauréline Seurre, Kätlin Rohtlaid, Giao T. M. Nguyen, Caroline Soyer, Sofiane Ghenna, Sébastien Grondel, Frederic Vidal, Barthelemy Cagneau, Cedric Plesse, Eric Cattan

\section{To cite this version:}

Lauréline Seurre, Kätlin Rohtlaid, Giao T. M. Nguyen, Caroline Soyer, Sofiane Ghenna, et al.. Demonstrating full integration process for electroactive polymer microtransducers to realize soft microchips. 33rd IEEE International Conference on Micro Electro Mechanical Systems (MEMS), Jan 2020, Vancouver, Canada. 10.1109/MEMS46641.2020.9056371 . hal-03322828

\section{HAL Id: hal-03322828 \\ https://hal.science/hal-03322828}

Submitted on 14 Sep 2021

HAL is a multi-disciplinary open access archive for the deposit and dissemination of scientific research documents, whether they are published or not. The documents may come from teaching and research institutions in France or abroad, or from public or private research centers.
L'archive ouverte pluridisciplinaire $\mathbf{H A L}$, est destinée au dépôt et à la diffusion de documents scientifiques de niveau recherche, publiés ou non, émanant des établissements d'enseignement et de recherche français ou étrangers, des laboratoires publics ou privés.

\section{다)(1) $(5$}

Distributed under a Creative Commons Attribution - NonCommerciall 4.0 International 


\title{
DEMONSTRATING FULL INTEGRATION PROCESS FOR ELECTROACTIVE POLYMER MICROTRANSDUCERS TO REALIZE SOFT MICROCHIPS
}

\author{
Lauréline Seurre ${ }^{1}$, Kätlin Rohtlaid ${ }^{2}$, Giao T. M. Nguyen ${ }^{2}$, Caroline Soyer ${ }^{1}$, Sofiane Ghenna ${ }^{1}$, \\ Sébastien Grondel ${ }^{1}$, Frédéric Vidal ${ }^{2}$, Barthélemy Cagneau ${ }^{3}$, Cédric Plesse $^{2}$, and Eric Cattan ${ }^{1}{ }^{*}$ \\ ${ }^{1}$ Univ. Polytechnique Hauts-de-France, CNRS, Univ. Lille, Yncrea, Centrale Lille, UMR 8520 - \\ IEMN, DOAE, Valenciennes, France \\ ${ }^{2}$ LPPI, EA2528, Institut des Matériaux, Université de Cergy-Pontoise, Cergy Cedex, France \\ ${ }^{3}$ Université de Versailles, UVSQ/LISV, France
}

\begin{abstract}
Actuation and sensing with electroactive polymers should be a chance for flexible MEMS but their micromachining and integration are still not mature. Some innovative materials and microfabrication processes are still expected. In this paper, a first full elaboration of polymeric microtransducers (MTs) including integration and operation has been described. The fabrication process relies on commercially available poly(3,4-ethyledioxythiophene):poly(styrene sulfonate) (PEDOT:PSS) conductive ink, onto flexible SU-8 photoresist microchip. Batch-fabrication of complex flexible monolithic units comprising individually addressable MTs of different shapes, is demonstrated. The resulting polymeric MTs show both very promising bending actuation and strain sensing properties in open-air. Remarkably, the microfabrication process did not impact the performances compared to material fabricated with laser cutting. This work paves the way for flexible MEMS development for soft microrobotics, microfluidics in medical and spatial applications.
\end{abstract}

\section{KEYWORDS}

Polymeric microtransducer, flexible microchip, electronically conducting polymer, PEDOT:PSS.

\section{INTRODUCTION}

The use of organic materials to develop flexible microelectronic devices such as electronic paper, touch screens, solar cells, and wearable electronics is now widely described. However, new functional materials, being actuators and/or sensors, are still expected to be developed and fully integrated into flexible MEMS. Polymeric actuators, such as electroactive polymers [1], electrothermal actuators [2], and magnetic actuators [3] are then increasingly studied to be used and potentially integrated into flexible microstructures. The mechanical sensing components also need to be considered since they allow feedback control. Materials with dual functionality should be thus favored to simplify the fabrication and integration processes, as only one material would have to be considered.

For flexible electronic circuits, electronic conducting polymers (ECPs) like commercially available poly $(3,4-$ ethylenedioxythiophene):polystyrene sulfonate (PEDOT: PSS), deposited with various techniques are widely studied to enhance its electronic conductivity. Moreover, ECPs have been also considered for their potential dimensional changes under electrical stimulation. This actuation functionality is generated by the ion expulsion/inclusion motions during an oxidation and reduction process in the presence of an electrolyte [4]. Additionally, they can operate in reverse mode as strain/force sensors, when mechanical stimulation is converted into an electrical response [5]. ECPs with a bilayer structure have been first studied. The integration of an ECP-based bilayer actuators operating only in an electrolyte liquid is published in 2000 s [6]. To operate in air, trilayer structures combining two ECPs sandwiched a supporting polymer membrane are fabricated. Ions necessary for redox process were introduced to the system by swelling in ionic liquid. Among supporting polymer used layers we can find polyvinylidene fluoride PVDF membrane [7], interpenetrating polymer networks (IPNs) like polytetrahydrofurane/poly(ethylene oxide) PTHF/PEO [8] or nitrile butadiene rubber/poly(ethylene oxide) NBR/PEO [9]. For these configurations, PEO promotes the high ionic conductivity while elastomer (NBR or PTHF) improves the mechanical properties of the middle matrix. However, to our knowledge, no result has been obtained so far for a fully integrated ionic trilayer electroactive polymer proving actuation and sensing capabilities. All the previous studies concerning the integration of ECP-based microactuators operating in air have been reported with manual handling steps $[10,11]$, without integrated remote electrical contacts $[11,12]$, and without a complete integration process [13].

Moreover, the most important advantages of these microtransducers (MTs) are: lightness, low cost and biocompatibility. They can be designed with complex shapes and can operate at low voltage (1-2 V). Besides, they are versatile since they can operate in liquid, in air and even in low pressurized environments. Because of all these advantages, they are promising candidates to enable a broad range of applications for a new generation of flexible microsystems. Large strains and forces with sensing capabilities are indeed required in several fields such as microrobotics and biomedical applications.

In this work, we present the fabrication of PEDOT:PSS-based MTs fully integrated into soft microchip and their characterization as actuator and sensor.

\section{MATERIAL AND INNOVATIVE PROCESS Materials}

The NBR/PEO IPN has been used as supporting polymer membrane. PEDOT:PSS commercially dispersion (Clevios PH1000) was chosen as an electrode material to fabricate MTs. Polyethylene glycol methacrylate derivatives (mPEG) has been used as reactive 
a)

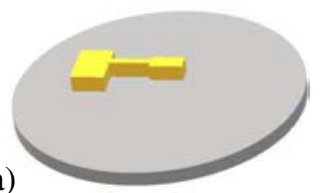

b)



c)

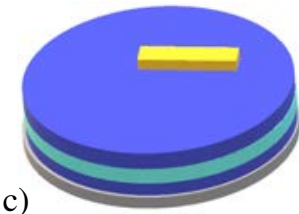

f)

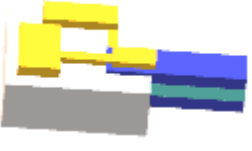

g)



d)

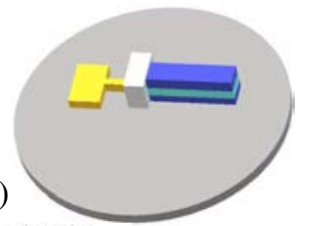

e)

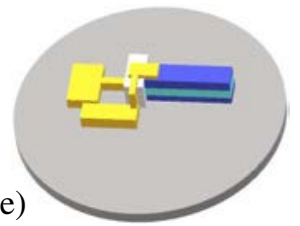

Electrodes

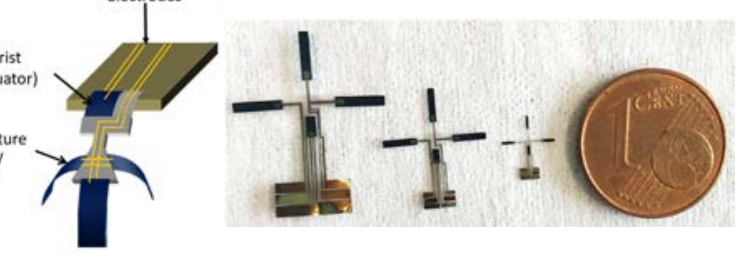

Silicon wafer $\square$ Gold

ECP PEDOT:PSS/PEO

Ion reservoir
NBR/PEO

Figure 1: Schematic representation of the main steps process for the integration of PEDOT:PSS-based actuator in soft structure. a) Au top electrode on Si wafer. b) Trilayer fabrication and Au evaporation. c) IBE of Au for hard mask. d)

RIE for trilayer pattern, then IBE of hard mask and SU-8 used as insulator. e) Au bottom electrode. f) SU-8

encapsulation for final flexible microchip and $\mathrm{XeF}_{2}$ vapor-phase etching of the Si wafer. The final prototype is the backside view of the previous step. g) Different prototypes incorporating conducting polymer-based MTs individually addressable in a flexible chip with gold remote contacts. h) Micro-grippers prototypes with three fingers and a wrist.

additive and directly incorporated into the PEDOT:PSS dispersion. $\mathrm{mPEG}$ has been chosen as additive because PEO based additives have been reported as an enhancement for electronic conductivity due to the morphological modifications of the conducting polymer layer. As a consequence, the electronic conductivity of the pristine PEDOT:PSS electrode is only $1{\mathrm{~S} . \mathrm{cm}^{-1}}^{-}$and reaches

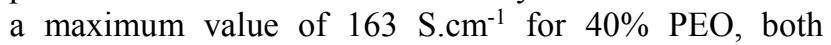
previously swollen in electrolyte. Moreover, the ionic conducting promotion of PEO phase allows for better ion transport and exchange between the electroactive PEDOT domains and the electrolyte 1-ethyl-3-methylimidazolium bis(trifluoro-methanesulfonyl)imide (EMImTFSI). Finally, the reactive nature of $\mathrm{mPEG}$ additives, via the methacrylate functions enhances the mechanical properties of the PEDOT:PSS electrodes and improves the adhesion between the electrodes and the ionic conducting membrane NBR/PEO due to covalent co-bonding. Final MTs are obtained after swelling the trilayers during $72 \mathrm{~h}$ in ionic liquid.

\section{Microfabrication of flexible chip including polymeric transducer}

To integrate ECP-based MTs into flexible chip, photolithography, gold evaporation and dry etching are used with 8 steps of mask. The prototypes are fabricated on 2 inches' double-side polished silicon substrates, with a thickness of $150 \mu \mathrm{m}$. A first gold electrode of $500 \mathrm{~nm}$ is obtained by evaporation and lift-off (Fig. 1a). The synthesis of the trilayer actuators is performed by stacking PEDOT:PSS/PEO, NBR/PEO and PEDOT:PSS/PEO layers with $8 \mu \mathrm{m}$ and $10 \mu \mathrm{m}$ thicknesses respectively, as described in [14] (Fig. 1b). A hard mask of gold layer is patterned to define the shape of the transducer etched with a reactive ion etching (RIE) using $\mathrm{O}_{2} / \mathrm{CF}_{4}$ gas at 90/10 ratio [15] (Fig. 1c). Then SU-8 photoresists $(2 \mu \mathrm{m})$ are used as insulator to avoid short-circuit between both layers of PEDOT:PSS/PEO (Fig. 1d), after the deposit of the bottom gold remote contact. Next, a last gold layer $(500 \mathrm{~nm})$ is deposited by evaporation for the bottom remote contact and patterned by ion beam etching (IBE) (Fig. 1e). To be manipulated, the microactuators are encapsulated with SU8 layer with a thickness defined as a function of the desired flexibility. Finally, the microactuators are released by etching all the silicon wafer, using vapor phase $\mathrm{XeF}_{2}$ etching cycles (Fig. 1f). Remote contacts address each MT in the same microchip for actuation and/or sensing. MTs $2 \mathrm{D}$ geometry can be tuned at will.

The originality of the manufacturing process lies in different aspects. Firstly, the process needs to consider the hygroscopicity of the PEDOT:PSS, meaning that no waterbased medium can be used after manufacturing the MT. Therefore, the negative SU-8 photoresist remains as the only usable photoresist. The wet chemical etching is avoided for the same reason. Secondly, a continuous electrical remote contact from the Si wafer to the top of the MTs, $26 \mu \mathrm{m}$ higher, is performed. Owing to an intermediate SU-8 insulating layer, no short circuits were observed on our microchips. Finally, the flexible microchips are provided with self-standing MTs fully integrated, individually addressable, with different shapes like combs (Fig. 1g), membranes, micro-grippers (Fig. 1h) and could be easily hybridized to another flexible microsystem.

\section{EXPERIMENTAL RESULTS \\ Young's modulus}

Stiffness measurements of the MT beams were carried out with the microrobotic system FT-RS1002 and a microforce sensing probe FT-S1000 (Femtotools). The tip of the sensing probe was in contact with a beam at the distance of $2 \mathrm{~mm}$ from the encapsulated base of the MT. The displacement was applied and the resulting force was recorded. The Young's modulus is calculated following the formula below:

$$
\mathrm{E}=4 k L^{3} /\left(w h^{3}\right)
$$

where $k$ is the slope of the recorded force and the displacement curve, $L$ is the distance between the clamped point of the beam and the tip of the force sensor, $w$ and $h$ 

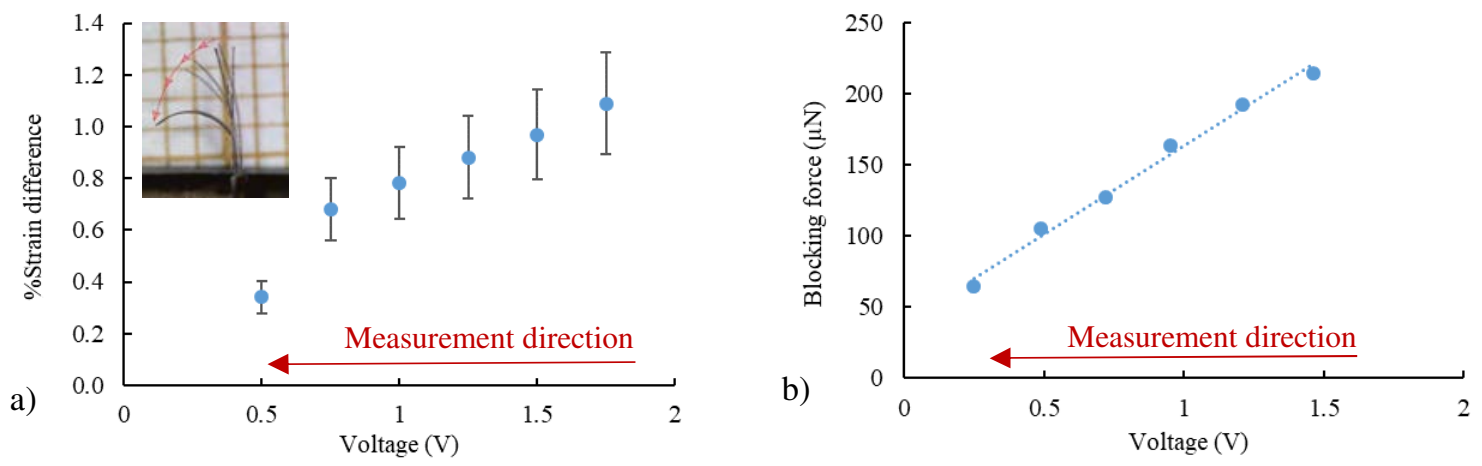

Figure 2: Measurements on one microbeam (width $728 \mu \mathrm{m}$, thickness $24.5 \mu \mathrm{m}$ ) of an integrated PEDOT:PSS-based microtransducer. a) \% strain difference and b) blocking force as a function of DC applied voltage from 1.5 to 0.25 $V$ (error bars are smaller than the point size). In insert: microbeam embedded in SU-8 actuated with a $V_{\text {in }}$ of $1.75 \mathrm{~V}$ applied on the remote gold contacts.

are the width and thickness of the beam respectively. The Young's modulus of the integrated MT is measured to be 3 GPa. This value can be compared to a nonintegrated MT which is $1 \mathrm{GPa}$ (cut by laser [14]), meaning that the process modifies its mechanical properties.

\section{Strain difference, blocking force and sensing}

The actuation characterizations were performed as a function of positive voltage $\left(\mathrm{V}_{\text {in }}\right)$ step during the required time to obtain the maximum of the displacement or the blocking force. At $1.5 \mathrm{~V}$ the rise time of the strain difference is more than $18 \mathrm{~min}(1131 \mathrm{~s})$. This value is explained by the very slow migration of ions. In comparison, for the same $V_{\text {in }}$, the rise time in blocking force is only of $108 \mathrm{~s}$. The ion migration continues beyond this time but the speed is so slow that it produces variations of forces lower than the sensitivity of our microforce sensing probe $(<\mu \mathrm{N})$. The figure 3 shows that rise time increases when the $\mathrm{V}_{\text {in }}$ decreases as expected.

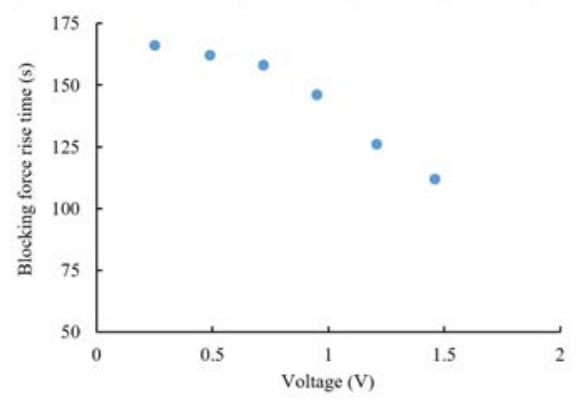

Figure 3: Blocking force rise time as function of the applied $V_{\text {in }}$ measured on a microbeam ( $w=728 \mu \mathrm{m}$, $h=24.5 \mu \mathrm{m})$ after full integration.

Beyond $1.75 \mathrm{~V}$, the degradation of the performances is observed in particular when $V_{\text {in }}$ is applied during a long time. The measurements are made from the highest $V_{\text {in }}$ to the lowest because of the memory effect of these materials. The performances depend on the previous state of the material.

The strain difference $\varepsilon$ was calculated according to Sugino et al equation [16] and reaches, linearly with $V_{\text {in }}$, the value of $1.1 \%$ at $1.75 \mathrm{~V}$ (Fig. 2a) for an integrated MT. For laser patterned microactuators (1374 $\mu \mathrm{m}$ of width), a value of $1.8 \%$ is reached for the same thickness, without integration step or integrated electrical connections. Moreover, the variations of the blocking force as function of $\mathrm{V}_{\text {in }}$ is linear from $0.5 \mathrm{~V}$ to $1.5 \mathrm{~V}$. The PEDOT:PSS-based MT beam demonstrated a maximum output force of 215 $\mu \mathrm{N}$ (Fig. 2b). These results prove that the microfabrication process does not extensively damage the resulting microbeams in comparison with MTs obtained by laser cutting without integration.

Moreover, sensing capabilities were evaluated by measuring the potential difference between the two ECPs of a microbeam after applying a strain. A high-precision three-axis piezoelectric manipulation unit with integrated optical encoders (FT-RS1002) is used to measure simultaneously the displacement and the force applied with the microforce sensing probe (Fig. 4a). A maximum output voltage of $0.4 \mathrm{mV}$ when a mechanical strain of $0.58 \%$ was applied (corresponding to a force of $400 \mu \mathrm{N}$ applied at 2 $\mathrm{mm}$ of the clamping point) (Fig. $4 \mathrm{~b}$ ). Such performance has never been achieved on so small and fully integrated MTs.

\section{CONCLUSION}

This paper proposes a successful microfabrication process to integrate small air operating PEDOT:PSS-based MTs for a flexible microchip. Integrated gold connections are in contact with the MT top and bottom electrodes to individually control each addressable MT. An innovative based on conventional techniques has demonstrated the integration of freestanding polymeric MTs with different shapes like combs, bridges, membranes. Microbeam actuation was successfully evaluated by a strain difference of $1 \%$ and a blocking force of $215 \mu \mathrm{N}$ at $1.5 \mathrm{~V}$ demonstrating the best achieved performance at this size. Moreover, sensing measurements were performed on the same microbeams, resulting in a maximum output voltage of $0.4 \mathrm{mV}$ with a mechanical strain of $0.58 \%$. These are the first measurements performed on such small microbeams. The flexibility of the microchips can be tuned by changing the thickness of the last SU-8 photoresist used during encapsulation. These microchips could be easily hybridized with other flexible or non-flexible microsystems such as microfluidic channels, parts of a micro and nanorobots or in optoelectronic components. 

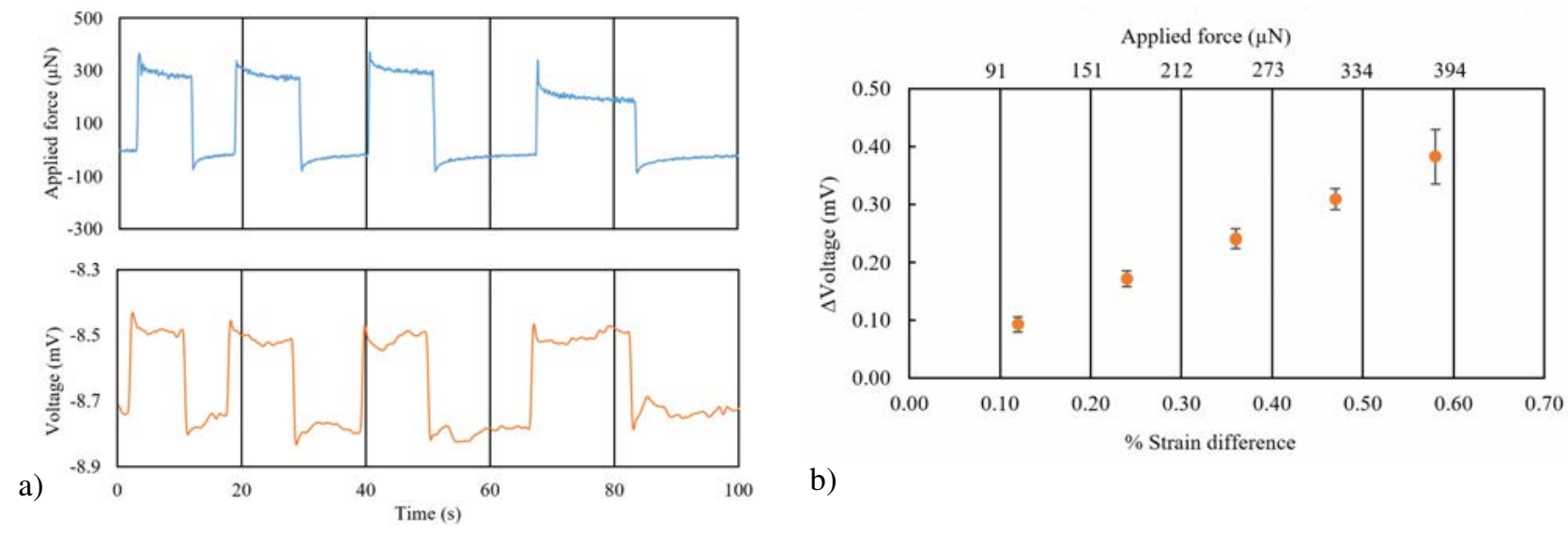

b)

Figure 4: a) Force applied on a microbeam for a mechanical displacement of $500 \mu \mathrm{m}$ and the measured sensing voltage response. b) Sensing voltage $(\Delta V)$ as function of strain difference (\%) or applied force $(\mu N)$. Measurement at $2 \mathrm{~mm}$ from the $\mathrm{SU}-8$ encapsulation.

\section{ACKNOWLEDGEMENTS}

This project received funding from the European Union's Horizon 2020 with project n ${ }^{\circ} 857263$ Twinnims. This work was partly financially supported by the French National Research Agency with PIA EQUIPEX LEAF (ANR-11-EQPX-0025), RENATECH and MicroTIP (ANR-15-CE08-0032-0) projects.

\section{REFERENCES}

[1] K. Jung, J. C. Koo, J. Do Nam, Y. K. Lee, H. R. Choi, "Artificial annelid robot driven by soft actuators", Bioinspiration and Biomimetics 2007, vol. 2, DOI 10.1088/1748-3182/2/2/S05.

[2] N. T. Nguyen, S. S. Ho, C. L. N. Low, J., "A polymeric microgripper with integrated thermal actuators" Micromechanics Microengineering 2004, vol. 14, 969.

[3] Z. Z. Chong, W. J. Sim, Z. T. Yeo, K. H. Li, S. H. Ng, H. Xia, T. N. Wong, N. H. Loh, S. B. Tor, S. H. Tan, N.-T. Nguyen, "Elastic magnetic membrane for improved mixing in microwells", Micro Nanosyst. 2014, vol. 6, 232.

[4] R. H. Baughman, "Conducting polymer artificial muscles", Synth. Met. 1996, 78, 339.

[5] Y. Wu, G. Alici, J. D. W. Madden, G. M. Spinks, G. G. Wallace, "Soft Mechanical Sensors Through Reverse Actuation in Polypyrrole", Adv. Funct. Mater. 2007, vol. 17, 3216.

[6] E. W. H. Jager, O. Inganäs, I. Lundström, "Microrobots for Micrometer-Size Objects in Aqueous Media: Potential Tools for Single-Cell Manipulation", Science 2000, vol. 288, 2335.

[7] A. Khaldi, A. Maziz, G. M. Spinks, E. W. Jager, "Bottom-up microfabrication process for individually controlled conjugated polymer actuators", Sensors and Actuators, B Chem, 2016, vol. 230, 818-824.

[8] C Plesse, A Khaldi, Q Wang, E Cattan, D Teyssié, C Chevrot and F Vidal "Polyethylene oxidepolytetrahydrofurane-PEDOT conducting interpenetrating polymer networks for high speed actuators", Smart Mater. Struct., 2011, vol. 20, 124002
[9] N. Festin, A. Maziz, C. Plesse, D. Teyssi'e, C. Chevrot and F. Vidal, "Robust solid polymer electrolyte for conducting IPN actuators", Smart Mater. Struct., 2013, vol. 22, 104005

[10] E. W. H. Jager, N. Masurkar, N. F. Nworah, B. Gaihre, G. Alici, G. M. Spinks, "Patterning and electrical interfacing of individually controllable conducting polymer microactuators", Sensors Actuators, B Chem. 2013, vol. 183, 283.

[11] A. Khaldi, A. Maziz, G. Alici, G. M. Spinks, E. W. H. Jager, "Bottom-up microfabrication process for individually controlled conjugated polymer actuators", Sensors Actuators, B Chem. 2016, vol. 230,818 .

[12] A. Maziz, C. Plesse, C. Soyer, E. Cattan, F. Vidal, "Top-down Approach for the Direct Synthesis, Patterning, and Operation of Artificial Micromuscles on Flexible Substrates", ACS Appl. Mater. Interfaces 2016, vol. 8, 1559.

[13] Y. Zhong, S. Lundemo, E. W. H. Jager, "Development of polypyrrole based solid-state onchip microactuators using photolithography", Smart Mater. Struct. 2018, vol. 27, 074006.

[14] K. Rohtlaid, G. T. M. Nguyen, C. Soyer, E. Cattan, F. Vidal, C. Plesse, "PEDOT:PSS/PEO electrodes with improved electrical and electro-chemical properties for soft microactuators and microsensors" $A d v$. Electron. Mater. 2019, vol. 5, 1800948,

[15] A. Khaldi, C. Plesse, C. Soyer, E. Cattan, F. Vidal, C. Legrand, and D. Teyssié, "Conducting interpenetrating polymer network sized to fabricate microactuators", Appl. Phys. Letters, 2011, vol. 98, 164101

[16] T. Sugino, K. Kiyohara, I. Takeuchi, K. Mukai, K. Asaka, "Actuator properties of the complexes composed by carbon nanotube and ionic liquid: The effects of additives" Sensors Actuators, B Chem. 2009, vol. 141, 179.

\section{CONTACT}

*E. Cattan, tel: +33 327511243 ; eric.cattan@uphf.fr 